\title{
OSCILLATION THEOREMS FOR SECOND ORDER NONLINEAR DIFFERENTIAL EQUATIONS
}

\section{LYNN ERBE ${ }^{1}$}

ABstract. The oscillatory and nonoscillatory behavior of the nonlinear second order differential equation (1) $x^{\prime \prime}+p(t) f(x)=0$ is related to that of $(2)_{\lambda} x^{\prime \prime}+\lambda p(t) x=0, \lambda>0$. Under certain conditions on $p(t)$ and $f(x)$ it is shown that all solutions of (1) are oscillatory if $(2)_{\lambda}$ is oscillatory for all $\lambda>0$. In contrast to most of the literature on this subject, no sign or integrability conditions on $p(t)$ are explicitly assumed.

Consider the second order nonlinear differential equation

$$
x^{\prime \prime}+p(t) f(x)=0
$$

where $p(t) \in C[0,+\infty)$ and $f(x) \in C^{(1)}(-\infty,+\infty)$, with

$$
f^{\prime}(x) \geqq \frac{f(x)}{x}>0 \quad \text { for } x \neq 0 .
$$

As a special case we have

$$
x^{\prime \prime}+p(t) x^{2 n+1}=0 .
$$

In case $p(t)$ is eventually positive, oscillation and nonoscillation criteria for (1) and (3) have been extensively developed. (See [1] and the bibliography therein for the nonlinear case. Willett in [2] has an extensive bibliography for the case when (1) is linear.) However, much less is known for the nonlinear case when $p(t)$ is allowed to be negative for arbitrarily large values of $t$. It is the purpose of this paper to relate the oscillatory behavior of (1) with that of the linear equation

$$
x^{\prime \prime}+\lambda p(t) x=0, \quad \lambda>0,
$$

which, presumably, is easier to handle. We shall restrict attention to solutions of (1) which exist on some ray $[T,+\infty)$ where $T$ may depend on the particular solution. We shall at various times assume that the following condition holds:

$$
\liminf _{t \rightarrow \infty} \int_{T}^{t} p(s) d s>0 \text { for all large } T .
$$

Received by the editors May 19, 1969.

AMS Subject Classifications. Primary 3442, 3445.

Key Words and Phrases. Second order nonlinear oscillation, boundedness, linear oscillation.

1 This research was supported by a University of Alberta Post-Doctoral Fellowship. 
For the case when $f(x)=x^{2 n+1}$, our main result generalizes a theorem due to Utz [4] who assumes $p(t) \geqq 0$ and a theorem due to Waltman [5] who has shown that all solutions of (3) oscillate provided the following condition holds:

$$
\int^{\infty} p(s) d s=+\infty
$$

For $n=0$ we have by the well-known Fite-Wintner Theorem (see [6]) that condition (6) implies all solutions of (3) oscillate. In fact, we see that $(4)_{\lambda}$ is oscillatory for all $\lambda>0$.

Lemma 1. Let $u(t)$ be a nonoscillatory solution of (1) on $[T,+\infty)$ and let condition (5) hold. Then for all large $t$ we have $u(t) u^{\prime}(t)>0$.

Proof. Assume, to be specific, that $u(t)>0$ for $t \geqq T_{1}, T_{1} \geqq T$. Obvious modifications are valid when $u(t)<0$. If the lemma is not true, then either $u^{\prime}(t)<0$ for all large $t$ or $u^{\prime}(t)$ oscillates. In the former case we may suppose that $T_{1}$ is sufficiently large so that

$$
\int_{T_{1}}^{t} p(s) d s \geqq 0 \quad \text { for } t \geqq T_{1}
$$

and $u^{\prime}(t)<0$ for $t \geqq T_{1}$. Hence, we have

$$
\begin{aligned}
\int_{T_{1}}^{t} p(s) f(u(s)) d s= & f(u(t)) \int_{T_{1}}^{t} p(s) d s \\
& -\int_{T_{1}}^{t} f^{\prime}(u(s)) u^{\prime}(s) \int_{T_{1}}^{s} p(\sigma) d \sigma d s \geqq 0, \quad t \geqq T_{1} .
\end{aligned}
$$

Now integrating (1) we have by (7) that $u^{\prime}(t) \leqq u^{\prime}\left(T_{1}\right)<0, t \geqq T_{1}$, which contradicts the fact that $u(t)$ is nonoscillatory.

If $u^{\prime}(t)$ oscillates, let $T_{n} \rightarrow+\infty$ be such that $u^{\prime}\left(T_{n}\right)=0$. For $t \geqq T_{1}$ we define

$$
v(t)=-u^{\prime}(t) / f(u(t)),
$$

and differentiating, we get

$$
v^{\prime}(t)=p(t)+w(t)
$$

where

$$
w(t)=(v(t))^{2} f^{\prime}(u(t)) \geqq 0, \quad t \geqq T_{1} .
$$

Since $v\left(T_{n}\right)=0$ we integrate (9) between $T_{n}$ and $T_{n+1}$ and sum on $n$ to get an immediate contradiction to (5). 
THEOREM 2. Let (4) $\lambda_{\lambda}$ be oscillatory and let $u(t)$ be a nonoscillatory solution of (1) with $u(t) u^{\prime}(t)>0$ for all $t \geqq T$. Then

$$
\lim _{t \rightarrow \infty} \frac{f(u(t))}{u(t)} \leqq \lambda .
$$

Proof. Let $g(x)=f(x) / x$. We note that condition (2) implies that the limit in (9) exists (possibly infinite). If the theorem is not true, we may assume $g(u(t)) \geqq \lambda$ for all $t \geqq T$. Let $z(t)$ be the solution of (4) satisfying $z(T)=0, z^{\prime}(T)=1$, and let $T_{1}>T$ be the first zero of $z^{\prime}(t)$ so that $z^{\prime}(t)>0$ on $\left[T, T_{1}\right)$. Then

$$
\int_{T}^{T_{1}}(g(u(t))-\lambda)\left(z^{\prime}\right)^{2} d t \geqq 0
$$

so integrating by parts we get

$$
\begin{aligned}
\int_{T}^{T_{1}}(g(u(t))-\lambda)\left(z^{\prime}\right)^{2} d t & \\
\quad & \lambda \int_{T}^{T_{1}} p z^{2}(g(u(t))-\lambda) d t-\int_{T}^{T_{1}} z z^{\prime} g^{\prime}(u(t)) u^{\prime} d t \\
& \leqq \lambda \int_{T}^{T_{1}} p z^{2}(g(u(t))-\lambda) d t
\end{aligned}
$$

since the integrand $z z^{\prime} g^{\prime}(u(t)) u^{\prime}$ is nonnegative on $\left[T, T_{1}\right]$ by condition (2). But

$$
\begin{aligned}
\int_{T}^{T_{1}} p z^{2}(g(u(t))-\lambda) d t \\
\quad=\int_{T}^{T_{1}} \frac{z}{u}(p z f(u(t))-\lambda p z u) d t \\
\quad=\int_{T}^{T_{1}} \frac{z}{u}\left(z^{\prime} u-u^{\prime} z\right)^{\prime} d t \\
\quad=-u^{\prime}\left(T_{1}\right)\left(z\left(T_{1}\right)\right)^{2} / u\left(T_{1}\right)-\int_{T}^{T_{1}}\left(\left(z^{\prime} u-u^{\prime} z\right) / u\right)^{2} d t<0,
\end{aligned}
$$

and this is a contradiction.

Lemma 1 along with Theorem 2 imply the following:

Corollary 3. Let (4) $\lambda_{\lambda}$ be oscillatory and assume condition (5) holds. Then all nonoscillatory solutions of (3) are bounded. In fact, if $u(t)$ is a nonoscillatory solution of (3), then 


$$
\lim _{t \rightarrow \infty}|u(t)|=\gamma \leqq(\lambda)^{1 / 2 n}
$$

Theorem 4. Assume (4)入 is oscillatory for all $\lambda>0$ and assume condition (5) holds. Then all solutions of (1) oscillate.

Proof. If not, let $u(t)$ be a nonoscillatory solution of (1). Lemma 1 and Theorem 2 imply $u(t)$ satisfies $u(t) u^{\prime}(t)>0$ for all large $t$ and $\lim _{t \rightarrow \infty} g(u(t))=0$. But this is a contradiction since $d(g(u(t))) / d t \geqq 0$ by (2). This proves the theorem.

Consider the following somewhat weaker condition than (5): There exists a sequence $T_{n} \rightarrow+\infty$ such that

$$
\int_{T_{n}}^{t} p(s) d s \geqq 0, \quad t \geqq T_{n} .
$$

The proof of Lemma 1 and Theorem 2 imply

CoRollary 5. If $p(t)$ satisfies condition $\left(5^{*}\right)$, and if $(4)_{\lambda}$ is oscillatory for all $\lambda>0$, then $u^{\prime}(t)$ oscillates for all solutions $u(t)$ of (1).

ExAmples. Willett [3] has shown that $(4)_{\lambda}$ is oscillatory for all $\lambda>0$ where $p(t)=t^{\eta} \sin t$ and $\eta>-1$. Thus, Corollary 5 implies that $u^{\prime}(t)$ oscillates for all solutions $u(t)$ of $(1)$ if $-1<\eta \leqq 0$.

For the equation

$$
x^{\prime \prime}+\left(\rho t^{-2}+\mu t^{-1} \sin \nu t\right) x=0
$$

results in [3] imply oscillation if $\rho>\frac{1}{4}-\frac{1}{2}(\mu / \nu)^{2}$ and nonoscillation if $\rho<\frac{1}{4}-\frac{1}{2}(\mu / \nu)^{2}$. Moreover, $p(t)$ satisfies condition (5) if $\rho>\mu / \nu \geqq 0$. Letting $\mu=\nu$ and $\rho>1$ it follows that $x^{\prime \prime}+\lambda p(t) x=0$ is oscillatory if $\lambda>\lambda_{0} \equiv\left(\rho^{2}+\frac{1}{2}\right)^{1 / 2}-\rho$ so that all nonoscillatory solutions of (3) satisfy $|u(t)| \leqq\left(\lambda_{0}\right)^{1 / 2 n}$ for all large $t$ by Corollary 3 .

\section{REFERENCES}

1. James S. W. Wong, On second order nonlinear oscillation, Funkcial. Ekvac. 11 (1969), 207-234.

2. D. Willett, Classification of second order linear differential equations with respect to oscillation, Advances in Math. 3 (1969), 594-623.

3. - On the oscillatory behavior of the solutions of second order linear differential equations, Ann. Polon. Math. 21 (1969), 175-194.

4. W. R. Utz, Properties of solutions of $u^{\prime \prime}+g(t) u^{2 n-1}=0$, Monatsh. Math. 66 (1962), 55-60. MR 25 \#2275.

5. Paul Waltman, An oscillation criterion for a nonlinear second order equation, J. Math. Anal. Appl. 10 (1965), 439-441. MR 30 \#3265.

6. A. Wintner, A criterion of oscillatory stability, Quart. Appl. Math. 7 (1949), 115-117. MR 10, 456.

University of Alberta 